\section{Predictors of HIV stigma among health workers in the Cape Coast metropolis, Ghana}

\author{
Prah James, ${ }^{1}$ \\ Anna Hayfron-Benjamine, ${ }^{2}$ \\ Mohammed Abdulai, ${ }^{1}$ \\ Obed Lasim, ${ }^{3}$ Nartey Yvonne, ${ }^{4}$ \\ Dorcas Obiri-Yeboah ${ }^{5}$ \\ ${ }^{1}$ University Hospital of Cape Coast, \\ University of Cape Coast; ${ }^{2}$ Department \\ of Nursing, School of Nursing and \\ Midwifery, College of Health \& Allied \\ Sciences, University of Cape Coast; \\ ${ }^{3}$ Department of Health Information \\ Management, College of Health \& Allied \\ Sciences, University of Cape Coast; \\ ${ }^{4}$ Department of Internal Medicine, Cape \\ Coast Teaching Hospital, Cape Coast; \\ ${ }^{5}$ Department of Microbiology and \\ Immunology, School of Medical \\ Sciences, College of Health \& Allied \\ Sciences, University of Cape Coast, \\ Ghana
}

\begin{abstract}
Stigma against people living with HIV (PLHIV) among health workers remains a barrier to the provision of needed care to HIV patients worldwide. We sought to investigate predictors of HIV stigma among health workers in Cape Coast, Ghana. This was a cross sectional study conducted in three hospitals from November 2016 to February 2017. The study made use of a pre-existing self-administered questionnaire. Multiple regression analysis and Pearson's coefficient tests were performed to determine the relationship between the dependent variable, i.e. the stigmatized attitudes of health workers, and some independent variables, and to determine which of the independent variables predict stigma amongst health care workers. A total of 331 health workers of different categories participated. Respondents' personal opinions on HIV, fears/worries of getting infected and category of staff $\{\mathrm{F}(3,327)=23.934$ $\left.\mathrm{P}<0.00\}, \mathrm{R}^{2}=0.180\right)$ significantly predicted their stigmatizing behaviour. Compared to other health workers, nurses had higher scores for stigmatizing behaviour. The study found evidence of discriminatory and stigmatizing behaviour towards PLHIV by health workers. Stigma reduction programmes aimed at health workers should be strengthened at all health facilities that provide care to PLHIV.
\end{abstract}

\section{Introduction}

Globally, it is widely accepted that stigma against people infected with Human Immunodeficiency Virus (HIV) continues to be a significant barrier to the response to combat the HIV epidemic. ${ }^{1}$

Comprehensive HIV care using antiretroviral therapy (ART) in Ghana began in June 2003. ${ }^{2}$ Over the years, the prevalence of HIV in Ghana has experienced a steady decline with occasional spikes. According to the 2017 Ghana HIV Sentinel Survey Report $^{3}$ the highest ever prevalence was $3.6 \%$, recorded in 2003, whilst the lowest prevalence has been $1.6 \%$, in 2014. The HIV prevalence in Ghana in 2017 was $2.1 \%$. The estimated number of PLHIV in Ghana in 2017 was 313,063. The prevalence in the Central Region of Ghana has remained stable over the last three years at $1.8 \%{ }^{3}$

According to Joint United Nations Program on HIV/AIDS (UNAIDS, 2003), HIV/AIDS related stigma can be described as a process of devaluation of people either living with or associated with HIV and AIDS. ${ }^{4}$ Stigma occurs when the negative social meanings attached to a discrediting attribute become linked to the person with or perceived to have the attribute. ${ }^{5,6}$ Such discrediting attributes that generates stigma have been found to include HIV. ${ }^{7}$ HIV-related discrimination, which is also known as enacted HIV stigma, is the unfair and unjust treatment of an individual based on his or her real or perceived HIV status.

Several studies have documented the negative effects of stigma on people living with HIV (PLHIV). These effects include low retention in care, delaying treatment or not adhering to treatment prescribed and even more worrying not adopting preventive measures such as using condoms when having intercourse with partners who may be HIV negative. ${ }^{8}$

Health professionals may deliberately minimise contact with, or care of PLHIV, deny or avoid treating such patients and even isolate PLHIV from other patients. ${ }^{9}$ Globally, it is estimated that only $75 \%$ of people living with HIV know their status. ${ }^{10}$ This could be attributed to the stigma associated with the disease and people are thus not desirous to know their HIV serostatus. ${ }^{11}$ Unfortunately, the health care facility, which is the very place PLHIV go to seek care, is one of the places where they encounter serious forms of stigmatization and discrimination. ${ }^{12}$ PLHIV often name health care workers as important sources of stigma. ${ }^{13}$ Even though there has been intense public education on HIV/AIDS for decades, there is still documented stigma
Correspondence: James Prah, University of Cape Coast Hospital, University of Cape Coast, Ghana.

E-mail: james.prah@ucc.edu.gh

Key words: Stigma, Discrimination, PLHIV, Cape Coast, Ghana

Acknowledgements: The authors wish to express their profound appreciation to all staff of Cape Coast Teaching Hospital, University of Cape Coast Hospital and the Cape Coast Metropolitan Hospital who voluntarily participated in this study.

Contributions: JP contributed to the conception of the research idea, designing study, data analysis, and paper drafting, DOY designed the research, analyzed data and provided critical revision of the paper. AHB, MA, and YN contributed to collection of data, and revision of paper. OL contributed to data analysis, interpretation of results and paper reviewing. All authors reviewed the final manuscript.

Conflict of interest: The authors declare that they have no conflict of interest.

Funding: None.

Ethics approval and informed consent: The University of Cape Coast Institutional Review Board gave ethical approval for the study. (Protocol ID: UCC/IRB/A/2016/12) Permission was obtained from the administrators of each hospital before study was conducted. All respondents gave written informed consent before they participated in the study.

Received for publication: 15 January 2019.

Revision received: 10 May 2019:

Accepted for publication: 1 August 2019.

This work is licensed under a Creative Commons Attribution NonCommercial 4.0 License (CC BY-NC 4.0).

CC Copyright: the Author(s),2020

Licensee PAGEPress, Italy

Journal of Public Health in Africa 2020; 11:1020 doi:10.4081/jphia.2020.1020

and discrimination in many health care facilities worldwide. ${ }^{14,15}$ Acts of stigma and discrimination by health workers have been varied. These include refusing PLHIV health care, ${ }^{16}$ forced sterilization of women who are HIV positive, ${ }^{17}$ differential treatment, testing and disclosure of HIV status without consent, and verbal abuse. ${ }^{18,19}$ Such stigmatizing behaviours from health care staff often result from judgements associating HIV infection with immoral behaviours, fear of contracting infection through contact, and an inadequate knowledge on stig- 
ma and the consequences of stigma. ${ }^{11,16}$

Many previous studies in Ghana have sought to explore AIDS stigma among the general populace, with a few focusing on health workers. A study by Mills (2003) which assessed the experiences of HIV positive women who received care in Accra and Agomanya reported that many of the women felt the need to hide their HIV status. ${ }^{20}$ Another study conducted among the general population in Ghana, showed that $12 \%$ of participants indicated they will change jobs if someone they work with becomes infected with HIV. ${ }^{21}$ An in-depth interview study conducted among health care workers in Cape Coast, Ghana revealed a general fear of infection among health staff who care for PLHIV. ${ }^{22}$ Even though Ghana has made some strides in the control of HIV/AIDS, these gains may be eroded if HIV stigma, especially by health workers, is not addressed. Despite the laws and policies in Ghana that have been made to protect PLHIV from stigma and discrimination, the canker still persists. These laws and policies include the Ghana AIDS Commission Act 2016 (Act 938) and the National Guidelines for the implementation of HIV counselling and testing, 2008..$^{23,24}$

There is, however, little knowledge of how the health care system influences stigma among health care workers and the existence of stigmatizing behaviour among health professionals towards PLHIV in Ghana. There is therefore a need for comprehensive information to inform policymakers, hence justifying the need for this study. This study therefore sought to assess the prevalence of stigma and identify factors associated with stigmatizing behaviour towards PLHIV among health workers in Cape Coast, Ghana.

\section{Materials and Methods}

\section{Study design}

This was an analytical cross sectional study conducted in three hospitals in the Cape Coast metropolitan area from November 2016 to February 2017.

\section{Study population}

The study involved health workers involved in HIV care in three hospitals in the Cape Coast Metropolis. The health workers were put in two main categories, clinical and non-clinical. Clinical staff includes those who, by the nature of their work, are likely to have physical contact with PLHIV. These include prescribers (doctors, dentists, physician assistants), diagnostic staff (laboratory staff, radiogra- phers), nurses and midwives (all categories). Non-clinical staff includes those who usually do not have physical contact with PLHIV, such as pharmacists, dispensing technicians, account workers, health information staff, catering staff and security men.

\section{Study sites}

The hospitals selected for the study were Cape Coast Teaching Hospital, University of Cape Coast Hospital and Metropolitan Hospital, Cape Coast. These hospitals provide full antiretroviral therapy and care for PLHIV. Services provided by these hospitals include counselling and testing, provision of antiretroviral drugs, conducts laboratory investigations needed in the management of clients and care of inpatients. The Cape Coast Teaching Hospital (about 400 beds) is the only tertiary level hospital and the largest specialist hospital that provides services to PLHIV in the Central and Western regions of Ghana. ART clinic began in the hospital in the year 2006 with currently about 2000 patients on ARVs. The University of Cape Coast Hospital ( 80 bed capacity facility) began provision of comprehensive HIV care in 2009 and currently has about 200 clients on ARVs. The Cape Coast Metropolitan Hospital (about 100 beds) is a primary level health facility that has been providing full ART services to PLHIV since 2009 with about 400 clients on ARVs currently.

\section{Sample size}

A minimum sample size of 384 was determined using the statistical formula of Fisher (1998) for calculating sample size. $\mathrm{N}=\mathrm{Z}^{2} \mathrm{pq} / \mathrm{d}^{2}$ where: $\mathrm{N}=$ Minimum sample size for a statistically significant survey; $\mathrm{Z}$ $=$ Normal deviant at the portion of $95 \%$, Confidence interval $=1.96 ; \mathrm{P}=$ Prevalence of $50 \%$ was used since there was no previous prevalence of stigmatizing behaviour among health workers in our setting, $\mathrm{q}=1$ $\mathrm{P} ; \mathrm{d}=$ Margin of error acceptable or measure of precision $=0.05$. A systematic $(3: 1)$ sampling technique was then used to recruit participants who met the inclusion criteria into the study. The first respondent was selected for each facility by balloting and picking a number between one and three.

\section{Inclusion criteria}

The study population included all health workers (clinical and non-clinical staff) who had at least one year work experience post qualification. Any health worker who worked outside the selected hospitals was excluded.

\section{Data collection instrument}

A pre-developed survey instrument that was used in an earlier study to determine the stigmatizing behaviour of health care staff in Ghana was adapted with some modifications. ${ }^{25}$ The modifications included removing some questions that were not applicable to our setting. The original research tool had been pretested in various regions of the world to ensure its empirical validity. ${ }^{26}$ The questionnaire covered socio-demographic characteristics of respondents, opinions of heath care workers about PLHIV, experience of direct contact with PLHIV, knowledge on HIV transmission and prevention.

\section{Data collection}

Data were collected using a self-administered questionnaire. The dependent variable in the study was the stigmatized attitudes of health workers. It was measured by a set of four items that reflects the respondents' and other health workers' avoidance behaviour towards PLHIV. The items were measured on a 4 point Likert-type scale. The total score of these 4 items ranges from 4 to 16 , with higher scores indicating more stigmatized attitudes toward PLHIV. The independent variables included personal opinions of health workers on PLHIV (this sought to assess whether health workers perceive PLHIV positively or negatively), fears or worries about HIV transmission, and knowledge about institutional policies on HIV.

Responses to questions posed in the questionnaire were assigned assumed scores as follows: on respondents' fears about HIV transmission, not worried $=1$, a little worried $=2$, worried $=3$, and very worried $=4$. Fear was defined as either being worried or very worried about the questions posed.

On respondents' personal opinions towards PLHIV and their knowledge on work place policies on HIV care, the following responses were scored as follows: strongly disagree $=1$, disagree $=2$, agree $=$ 3 , and strongly agree $=4$. Personal opinions were categorized as negative if a respondent either agreed or strongly agreed with the statements provided.

With regards to the enacted and observed stigmatizing behaviour shown by health workers, the following assumed scores were assigned to these responses: never $=1$, sometimes $=2$, most of the time $=3$, and all the time $=4$.

None of the scored questions were weighted. Scores obtained by each respondent for each question were summed and mean scores determined by dividing total score obtained for each question by 4 (number of responses). 


\section{Data analysis}

Data collected were coded and entered into SPSS version 20. Descriptive analysis was done using frequencies for demographic, professional data and responses to the questions posed. Multiple regression analysis and Pearson's coefficient were used to determine the relationship between the dependent variable and the independent variables, and to determine which of the independent variables predict stigma amongst health care workers. One-way ANOVA was used to compare stigma scores across the different categories of health workers (doctors, nurses, diagnostic staff, pharmacy staff, physician assistance and other staff). This was followed by Tukey's HSD (honest significant difference) test. A 95\% confidence level was applied.

\section{Ethics approval and consent to par- ticipate}

The University of Cape Coast Institutional Review Board gave ethical approval for the study. (Protocol ID: UCC/IRB/A/2016/12) Permission was obtained from the administrators of each hospital before study was conducted. All respondents gave written informed consent before they participated in the study.

\section{Results}

Out of a total of 390 questionnaires distributed, 352 were returned, with response rate of $90.3 \%$. Twenty-one questionnaires were not included in the analysis because of large missing data. Thus, data obtained from 331 respondents from different categories of health professionals were analysed after cleaning the data. Table 1 shows the socio-demographic characteristics of respondents. The mean age of respondents was $31.8 \pm 7.9$ years (range: $20-67$ years). Majority of respondents were nurses (161, $48.6 \%)$, followed by doctors $(48,14.5 \%)$ and laboratory staff $(25,7.6 \%)$. The others category comprised of catering staff, receptionists and security men. Women formed majority $(57.1 \%)$ of respondents. About $74.9 \%$ were clinical staff and $25.1 \%$ nonclinical staff. Participants had spent an average of $6.5 \pm 6.2$ years (range: $1-39$ years) in healthcare. About $55.6 \%$ of respondents had worked in a clinic that specialized in HIV care. Most of the respondents $(76.1 \%)$ were sure they had attended to a person living with HIV in the 3 months prior to the study. Most participants $(85.8 \%)$ said they had received some training in HIV care. On respondents' knowledge on HIV transmission, $51.4 \%$ correctly knew the percentage of HIV transmission globally that can be attributed to workplace exposure.

A multiple linear regression was calculated to predict stigmatizing behaviour of participants on their personal opinion on HIV clients, their fear of getting infected and the category of staff they belong. A significant equation was found $\{\mathrm{F}(3,327)=$ $23.934 \mathrm{P}<0.00$, with $\mathrm{R}^{2}$ of 0.180 . Participant's predicted their stigmatizing behaviour as equal to $1.664+0.155$ (fear of getting infected) +0.211 (personal opinions on HIV clients) + 3.824 (category of staff of respondent) as shown in Table 2 . Participants' average stigmatized behaviour increased 0.155 for each fear expressed, 0.211 for each negative opinion expressed and by 3.824 if he was a clinical staff. However, healthcare workers' knowledge on their health facilities policies on HIV, years of working in healthcare, and sex of respondents did not significantly predict their stigmatizing behaviour. An analysis of variance on stigmatizing scores yielded sig-

\section{Dependent variable: enacted stigma- tizing behaviour}

With regards to enacted behaviour, participants reported either most of the time or all the time engage in the following stigmatizing behaviours: they wear gloves for all contact when caring for PLHIV (61.3\%), they use other measures which they usually do not use for a other patients when caring for PLHIV (24.0\%), they try to avoid contact when providing care for a PLHIV $(17.8 \%)$, and wearing double gloves when providing care for PLHIV (17.4\%).

Respondents had observed the following stigmatizing behaviour either most of the time or all the time: observed colleagues used additional measures in caring for a PLHIV (43.6\%), health workers performing

Table 1. Socio-demographic characteristics and other variables related to HIV care of respondents $(\mathrm{N}=331)$.

$\begin{array}{ccc}\text { Age group (years) } & & \\ <26 & 62 & 18.7 \\ 26-40 & 228 & 68.9 \\ 41-55 & 30 & 9 . \\ >55 & 11 & 3.3\end{array}$

\begin{tabular}{lll} 
Gender & & \\
Male & 142 & 42.9 \\
Female & 189 & 57.1 \\
\hline
\end{tabular}

Category of staff

Nurses (all categories) $\quad 161 \quad 48.6$

Doctors (all categories) $\quad 48 \quad 14.5$

Diagnostic staff

Pharmacy staff

Physician assistants

Others

mer of years of practice

$\begin{array}{lcc}1-5 & 197 & 59.5 \\ 6-10 & 77 & 23.3 \\ 11-15 & 30 & 9.1 \\ 16-20 & 14 & 4.2 \\ >20 & 13 & 3.9\end{array}$

Ever worked in a clinic specialized in HIV care?

Yes $\quad 184$

$\begin{array}{lll}\text { No } & 147 & 44.4\end{array}$

\begin{tabular}{ccc} 
Average number of HIV patients cared for in the past 12 months $(\mathrm{n}=296)$ & \\
$<10$ & 165 & 55.7 \\
$10-50$ & 92 & 31.1 \\
$>50$ & 39 & 13.2 \\
\hline
\end{tabular}

Training on HIV care

$\begin{array}{lll}\text { Yes } & 284 & 85.8\end{array}$

$\begin{array}{lll}\text { No } & 47 & 14.2\end{array}$

\begin{tabular}{lcc} 
Percentage of global HIV transmission due to workplace exposure & \\
$<5$ & 170 & 51.4 \\
$5-15$ & 123 & 37.2 \\
$>15$ & 23 & 6.9 \\
Don't know & 15 & 4.5 \\
\hline
\end{tabular}


HIV test on a patient without consent $(22.0 \%)$, an HIV positive woman being asked to use family planning as a condition for providing antiretroviral drugs (13.7\%), other colleagues unwilling to care for a patient thought to be PLHIV (13.5\%), health workers speaking negatively about PLHIV (9.8\%) and health workers neglecting a PLHIV in labour (1.9\%) (Table 3).

Table 4 shows a summary of respondents' fears/worries about HIV transmission. Health workers expressed their fears regarding HIV transmission as either worried or very worried when performing surgery for a PLHIV (64.3\%), delivering a baby for a PLHIV (58.4\%), drawing blood from a PLHIV (48.7\%), dressing the wound of a HIV client (44.0\%), taking the temperature of a PLHIV (32.4\%), fear of being discriminated for caring for PLHIV $(27.0 \%)$, fear for people saying negative things about them taking care of PLHIV (25.0\%).

Majority $(52.8 \%)$ of non-clinical staff were worried about a health worker contracting HIV compared to clinical staff $(24.4 \%)$. About $71.9 \%$ of non-clinical staff was worried about getting HIV if a health worker drew blood from a PLHIV as com- pared to clinical staff $(52.0 \%)$. Many nonclinicians $(74.2 \%)$ were worried about a worker getting infected with HIV when he performs surgery. About $58.3 \%$ of clinicians shared similar worries. A higher proportion of clinicians $(37.6 \%)$ was worried about contracting HIV through wound dressing compared to non-clinicians $(34.8 \%)$. A minority of both clinicians $(20.2 \%)$ and non-clinicians $(34.0 \%)$ were worried about negative sayings about them for taking care of PLHIV.

Participants were asked questions on their knowledge of their health facilities'

Table 2. Regression analysis of variables predicting enacted stigmatizing behaviour of respondents.

\begin{tabular}{lccc} 
Independent variable & Unstandardived coefficient & Standard error & p-value \\
Category of staff & 3.824 & 0.501 & 0.000 \\
Opinions on HIV clients & 0.211 & 0.070 & 0.003 \\
\hline Fear of getting infected & 0.155 & 0.061 & 0.022 \\
\hline
\end{tabular}

Constant: $1.664: \mathrm{R} 2=0.180 ; \mathrm{SEE}=6.294 ; \mathrm{F}=23.934 ; \mathrm{n}=331$.

Table 3. Enacted and observed stigmatizing behaviour shown by health workers.

\begin{tabular}{|c|c|c|c|c|}
\hline Parameter & $\begin{array}{l}\text { Never } \\
(n, \%)\end{array}$ & $\begin{array}{l}\text { Sometimes } \\
(\mathrm{n}, \%)\end{array}$ & $\begin{array}{l}\text { Most of the time } \\
(\mathrm{n}, \%)\end{array}$ & $\begin{array}{l}\text { All the time } \\
(\mathrm{n}, \%)\end{array}$ \\
\hline \multicolumn{5}{|l|}{ Enacted stigma } \\
\hline $\begin{array}{l}\text { Do you use any measures which you don't use for other patients when } \\
\text { you are providing services for a PLHIV? }(\mathrm{N}=308)\end{array}$ & $96(31.2)$ & $138(44.8)$ & $37(12.0)$ & $37(12.0)$ \\
\hline Do you try to avoid physical contact when providing care or services for a PLHIV? $(\mathrm{N}=315)$ & $119(37.8)$ & $140(44.4)$ & $28(8.9)$ & $28(8.9)$ \\
\hline Do you wear gloves for all contact when providing care or services for a PLHIV? (N=313) & $36(11.5)$ & $85(27.2)$ & $66(21.1)$ & $126(40.2)$ \\
\hline Do you wear double gloves when providing care or services for a PLHIV? $(\mathrm{N}=311)$ & $124(39.9)$ & $133(42.7)$ & $21(6.7)$ & $33(10.7)$ \\
\hline \multicolumn{5}{|l|}{ Observed stigma } \\
\hline $\begin{array}{l}\text { In the past } 12 \text { months, how often have you observed healthcare workers } \\
\text { unwilling to care for a patient thought to be living with HIV }(\mathrm{N}=327)\end{array}$ & $192(58.7)$ & $91(27.8)$ & $34(10.4)$ & $10(3.1)$ \\
\hline $\begin{array}{l}\text { In the past } 12 \text { months, how often have you observed health workers speaking negatively } \\
\text { about a patient living with or thought to be living with HIV in your facility }(\mathrm{N}=328)\end{array}$ & $194(59.1)$ & $102(31.1)$ & $27(8.2)$ & $5(1.6)$ \\
\hline $\begin{array}{l}\text { In the past } 12 \text { months, how often have you observed health workers performing } \\
\text { a HIV test on a patient without informed consent }(\mathrm{N}=327)\end{array}$ & $198(60.6)$ & $57(17.4)$ & $37(11.3)$ & $35(10.7)$ \\
\hline $\begin{array}{l}\text { In the past } 12 \text { months, how often have you observed health workers neglecting } \\
\text { during labour and delivery because of her HIV status ( } \mathrm{N}=324)\end{array}$ & $294(90.7)$ & $24(7.4)$ & $6(1.9)$ & $0(0.0)$ \\
\hline $\begin{array}{l}\text { In the past } 12 \text { months, how often have you observed health workers using } \\
\text { additional measures in caring for a patient due to the HIV status }(\mathrm{N}=325)\end{array}$ & $98(30.2)$ & 85 (26.2) & $84(25.8)$ & $58(17.8)$ \\
\hline $\begin{array}{l}\text { Have you ever observed an HIV positive woman being asked to use family planning } \\
\text { if she wants to receive Anti-retroviral drugs }(\mathrm{N}=322)\end{array}$ & $232(72.0)$ & 46 (14.3) & $28(8.7)$ & $16(5.0)$ \\
\hline
\end{tabular}

Table 4. Respondents' fears/worries about HIV transmission.

\begin{tabular}{|c|c|c|c|c|}
\hline Question & $\begin{array}{l}\text { Not } \\
\text { worried } \\
(n, \%)\end{array}$ & $\begin{array}{l}\text { A little } \\
\text { worried } \\
(n, \%)\end{array}$ & $\begin{array}{l}\text { Worried } \\
\text { worried } \\
(n, \%)\end{array}$ & $\begin{array}{l}\text { Very } \\
\text { worried } \\
(n, \%)\end{array}$ \\
\hline How worried would you be about getting HIV if you dressed the wounds of a PLHIV? (N=277) & $78(28.2)$ & $77(27.8)$ & $35(12.6)$ & $87(31.4)$ \\
\hline How worried would you be about getting HIV if you drew blood from a PLHIV? (N=288) & $69(23.9)$ & $79(27.4)$ & $34(11.8)$ & $106(36.9)$ \\
\hline How worried would you be about getting HIV if you took the temperature of a PLHIV? (N=320) & $180(56.3)$ & $36(11.3)$ & $19(5.9)$ & $85(26.5)$ \\
\hline How worried would you be about getting HIV if you delivered the baby of a PLHIV? (N=325) & $62(19.1)$ & $73(22.5)$ & $55(16.9)$ & $135(41.5)$ \\
\hline How worried would you be about getting HIV if you performed surgery for a PLHIV? (N=322) & $47(14.6)$ & $68(21.1)$ & $46(14.3)$ & $161(50.0)$ \\
\hline How worried are you about people saying negative things about you for taking care of PLHIV? (N=325) & $214(65.8)$ & $30(9.2)$ & $20(6.2)$ & $61(18.8)$ \\
\hline Are you worried about your family and friends avoiding you for taking care of PLHIV? (N=326) & $189(58.0)$ & $46(14.1)$ & $27(8.3)$ & $64(19.6)$ \\
\hline
\end{tabular}


policies on HIV care. Their responses are shown on Table 5. Respondents strongly agreed on the following: it is not acceptable to perform HIV test on a patient without consent (44.2\%), their health facilities had a post exposure prophylaxis protocol to protect health workers $(35.2 \%)$, that there are adequate supplies and measures in their facilities to reduce the risk of workers becoming infected with HIV (18.7\%) and their facilities have written guidelines to protect PLHIV from discrimination $(9.3 \%)$.

With regards to their personal opinions on PLHIV, health workers strongly agreed to the following statements: women living with HIV should be allowed to have babies if they wish (34.8\%), pregnant women who refuse HIV test are irresponsible (18.9\%), most PLHIV do not care if they infect other people $(16.0 \%)$, if a pregnant woman is HIV positive, her husband has a right to know (14.4\%), women living with HIV should not get pregnant if they already have children (13.4\%), PLHIV have had many sexual partners $(6.7 \%)$, PLHIV should feel ashamed of themselves (5.9\%) and it can be appropriate to sterilize a woman living with HIV, even if this is not her choice $(5.9 \%)$ (Table 6).

\section{Discussion}

This study was conducted to assess the prevalence of stigma and to identify factors associated with stigmatizing behaviour towards PLHIV among health workers in Cape Coast, Ghana.

The study revealed that health workers' stigmatizing behaviour was influenced by their fears of getting infected with HIV in the course of their work, their negative opinions about PLHIV as well as the category of staff they belong. Many health workers were very worried about contracting HIV whilst performing surgeries on PLHIV with a minority very worried about people saying negative things about them for providing care for HIV clients. Even though most health workers did not report stigmatizing behaviour towards PLHIV, many of them had shown various forms of stigmatization towards PLHIV. Most participants had not observed stigmatizing behaviour on PLHIV by colleagues, however some of them had seen various forms of stigmatizing behaviour shown to HIV positive patients. Most respondents reported the presence of post exposure prophylaxis protocols aimed at protecting health workers in their facilities. Majority of health workers in the study reported that there were no written guidelines to protect PLHIV from discrimination in their facilities.

The finding that majority of participants in this study were females $(57.1 \%)$ and nurses $(48.6 \%)$ is consistent with that of a previous study in Ghana. ${ }^{25}$ In Ghana, majority of health workers are females and are mostly nurses. ${ }^{27}$ This study did not significantly relate gender to stigmatizing behaviours among health workers. This is inconsistent with the findings of some previous studies, ${ }^{28,29}$ that found that female health workers show more stigmatizing behaviour compared to their male counterparts. Nurses scored higher for stigmatizing behaviour compared to other health professionals. This finding agrees with a previous study, ${ }^{30}$ but in contrast to a Nigerian study ${ }^{31}$ that found that HIV stigmatizing attitude was highest among medical doctors compared to other categories of staff. A study conducted in five African countries ${ }^{32}$ found a high level of stigma among nurses towards PLHIV. Since nurses are usually the frontline staffs who provide very important health care services to HIV patients, such findings are worrying. Several

Table 5. Knowledge of respondents of health facilities' policies on HIV care.

\begin{tabular}{|c|c|c|c|c|}
\hline Statement & $\begin{array}{l}\text { Strongly } \\
\text { disagree } \\
(n, \%)\end{array}$ & $\begin{array}{l}\text { Disagree } \\
(\mathrm{n}, \%)\end{array}$ & $\begin{array}{l}\text { Agree } \\
(\mathrm{n}, \%)\end{array}$ & $\begin{array}{l}\text { Strongly } \\
\text { agree } \\
(n, \%)\end{array}$ \\
\hline In my facility it is not acceptable to test a patient for HIV without their knowledge $(\mathrm{N}=326)$ & 48 (14.7) & $37(11.3)$ & $97(29.8)$ & $144(44.2)$ \\
\hline I will get in trouble at work if I discriminate against PLHIV (N=323) & $27(8.4)$ & $38(11.8)$ & $152(47.0)$ & $106(32.8)$ \\
\hline $\begin{array}{l}\text { There are adequate supplies/measures in my facility that reduce my risk of becoming } \\
\text { infected with HIV }(\mathrm{N}=326)\end{array}$ & $38(11.70)$ & $82(25.1)$ & $145(44.5)$ & $61(18.7)$ \\
\hline $\begin{array}{l}\text { My health facility has written guidelines to protect patients living with HIV from } \\
\text { discrimination }(\mathrm{N}=321)\end{array}$ & $69(21.5)$ & $172(53.6)$ & $50(15.6)$ & $30(9.3)$ \\
\hline My health facility has post exposure prophylaxis protocol to protect health work & $32(10.0)$ & $42(13.1)$ & $134(41.7)$ & $113(35.2)$ \\
\hline
\end{tabular}

Table 6. Personal opinions of respondents towards PLHIV.

\begin{tabular}{|c|c|c|c|c|}
\hline Statement & $\begin{array}{l}\text { Strongly } \\
\text { disagree } \\
(n, \%)\end{array}$ & $\begin{array}{c}\text { Disagree } \\
(\mathrm{n}, \%)\end{array}$ & $\begin{array}{l}\text { Agree } \\
(\mathrm{n}, \%)\end{array}$ & $\begin{array}{l}\text { Strongly } \\
\text { agree } \\
(\mathrm{n}, \%)\end{array}$ \\
\hline Most PLHIV do not care if they infect other people (N=325) & $36(11.1)$ & $120(36.9)$ & $117(36.0)$ & $52(16.0)$ \\
\hline PLHIV should feel ashamed of themselves $(\mathrm{N}=326)$ & $173(53.1)$ & $105(32.2)$ & $29(8.8)$ & $19(5.9)$ \\
\hline PLHIV have had many sexual partners $(\mathrm{N}=326)$ & $122(37.4)$ & $132(40.5)$ & $51(15.4)$ & $21(6.7)$ \\
\hline People get infected with HIV because they engage in irresponsible behaviours ( $\mathrm{N}=324)$ & $102(31.3)$ & $130(39.3)$ & $79(23.9)$ & $15(5.5)$ \\
\hline Women living with HIV should be allowed to have babies if they wish ( $\mathrm{N}=326)$ & $22(6.8)$ & $43(13.3)$ & $146(45.1)$ & $113(34.8)$ \\
\hline $\begin{array}{l}\text { If a pregnant woman is HIV positive, her husband has a right to know, so I have a right } \\
\text { to tell him }(\mathrm{N}=326)\end{array}$ & $87(26.7)$ & $102(31.3)$ & $90(27.6)$ & $47(14.4)$ \\
\hline Pregnant women who refuse HIV testing are irresponsible $(\mathrm{N}=328)$ & $54(16.5)$ & $105(32.0)$ & $107(32.6)$ & $62(18.9)$ \\
\hline Women living with HIV should not get pregnant if they already have children $(\mathrm{N}=328)$ & $60(18.3)$ & $134(40.9)$ & $90(27.4)$ & $44(13.4)$ \\
\hline It can be appropriate to sterilize a woman living with HIV, even if this is not her choice (N=326) & $152(46.6)$ & 125 (38.3) & $30(9.2)$ & $19(5.9)$ \\
\hline
\end{tabular}


researchers have tried to find the reasons why nurses demonstrate stigma towards PLHIV. Some of the reasons identified are lack of in-depth knowledge on HIV and fear of being infected with HIV.

The number of years of working as health workers did not significantly predict their stigmatizing behaviour. This finding is consistent with a study conducted among Nigerian health workers ${ }^{31}$ and inconsistent with a study conducted among Chinese health workers ${ }^{31}$ that revealed that more experienced health workers showed less discrimination at work compared with their less experienced colleagues.

The fears and worries of health workers were found to be significant predictors of their stigmatizing behaviour. This is consistent with what was found in some earlier studies. ${ }^{16,25}$ These studies found that health worker's fears and misconceptions about how HIV is transmitted affects how they care for PLHIV. A number of respondents in this study for example were worried that they could get infected with HIV if they dressed the wound and drew blood from PLHIV. Such worries will certainly affect how these health workers care for their clients. Some fears and worries of health workers are actually misplaced as they are based on inadequate knowledge. For example, some respondents were worried they could acquire HIV just by taking the temperature of PLHIV. Such fears and worries about HIV transmission lead health workers to take unnecessary and often stigmatizing actions against PLHIV. The study found that many non-clinical staff expressed more fears about HIV transmission compared to their clinical counterparts. This could be due to the fact that clinicians receive more training on HIV transmission and how to protect themselves from getting infected compared to non-clinical staff.

The study found that chances of a health worker engaging in a stigmatizing behaviour towards PLHIV increased if he was a member of the clinical staff. This is because only activities performed by clinical staff were used to assess enacted stigma, most non-clinicians did not answer the questions posed since they were non applicable to them.

A study by Sayles et al. ${ }^{33}$ found that because of fear of getting HIV infection, health workers were wearing double gloves during procedures and were putting on face masks when taking blood pressures. Majority of respondents in this study affirmed that they used extra protective measures when providing routine services to PLHIV whilst others tried to avoid physical contact when providing care for PLHIV. These actions are stigmatizing and discriminating and could cause PLHIV not to patronize health facilities for needed care. Some respondents reported in this study that they had observed some stigmatizing behaviours towards PLHIV in their places of work. These observed behaviours included health workers performing HIV test on patients without their consent. These observed acts by health workers are in clear violation of the Ghana AIDS Commission Act, 2016, Act $938^{23}$ and the 2008 National Guidelines for the Implementation of HIV Counselling and Testing in Ghana. ${ }^{24}$ This law and policy both give the right to all to refuse or withdraw from a medical intervention including blood testing at any time. Thus in Ghana, HIV testing must be informed and voluntary. Some health workers may continue to engage in such acts unaware of the existence of such laws and the fact that victims may resort to legal proceedings against them.

In a Nigerian study, ${ }^{34} 80 \%$ of respondents who were PLHIV reported that their consent was not sought before HIV tests were conducted on them. This practice, they said, resulted in feelings of fear, shock and embarrassment upon hearing of their HIV status. Opinions of respondents' on PLHIV were found to significantly predict stigmatizing behaviour. Many respondents were of the opinion that PLHIV should be ashamed of themselves and that PLHIV got infected because they engaged in irresponsible behaviours. Similar findings were made among health workers in other parts of the world. ${ }^{28,35}$ Some respondents in this study disagreed with the statements there are adequate supplies/measures in my facility that reduce my risk of becoming infected with $H I \mathrm{~V}$ and my health facility has post exposure prophylaxis protocol to protect health workers. Such responses reflect the lack of clear-cut policies in some health facilities that are aimed at protecting both the care giver and the patient. Generally, it can be assumed that policies are needed to change behaviour. Nyblade et al. ${ }^{16}$ found that lack of specifying policies related to the provision of care to PLHIV can reinforce stigmatizing behaviour among health workers.

\section{Conclusions}

This study has brought to the fore many enacted and observed stigmatizing behaviours among health workers as well as the negative opinions many care givers have about PLHIV in our setting. An important lesson obtained from this study is that, in order to reduce stigma towards PLHIV at health facilities, interventions should focus on the individual health worker, the work- ing environment and policies at the work place. Stigma reduction programmes should be instituted at all health facilities that provide care to PLHIV. These should include education of health workers that adequately addresses their fears about transmission of HIV. At the work places, stigma reduction programmes should ensure that all logistics necessary for the practice of standard precautions are available. Drugs needed for post exposure prophylaxis should be available and easy to access. These may increase the confidence of health workers and let them avoid resorting to stigmatizing behaviours so as to protect themselves from occupational exposure to HIV. There should be avenues for PLHIV to report any stigmatizing and discriminating behaviours against them at all health facilities. In Ghana, a web-based mechanism for PLHIV to report discrimination that occurs in health care, education, employment and other areas has been developed by the Ghana AIDS Commission, the Commission on Human Rights and Administrative Justice and the Health Policy Project. ${ }^{37}$

Future studies should be aimed at developing interventions to address HIV related stigma among health workers.

\section{Limitations of the present study}

The main limitation to this study was the cross sectional nature of the study. Even though it could be used to make inferences about associations found in the study, it cannot be used to draw conclusions on cause and effect. Another limitation is that responses obtained may be biased towards socially acceptable behaviour at the work place instead of actual practices.

\section{References}

1. Link BG, Phelan JC. Stigma and its public health implications. The Lancet. 2006;367:528-9.

2. National Guidelines for Antiretroviral therapy- Ghana. (2016). Available from: https://aidsfree.usaid.gov/sites/default/f iles/gh_guidelines_arv_therapy.pdf. Accessed on October 2018

3. Ghana Health Services, Ministry of Health, NACP. National HIV Prevalence \& AIDS Estimates 20172022. Accra: 2018.

4. Ogden J, Nyblade L. HIV-related stigma across contexts: common at its core. International Center for Research on Women (ICRW): 2005 Available from: https://www.icrw.org/wp-content/ uploads/2016/10/Common-at-its-CoreH I V-Related-Stigma-Across- 
Contexts.pdf Accessed on October 2018

5. Berger BE, Ferrans CE, Lashley FR. Measuring stigma in people with HIV: Psychometric assessment of the HIV stigma scale. Res Nurs Health 2001;24: 518-29.

6. Health Policy Initiative. HIV stigma: A health policy approach. 2010. Available from: http://www.healthpolicyinitiative.com/Publications/Documents?125 7_1_EOP_Brief_HIV_Stigma_Health Policy_Approach_FINAL_Sep.pdf

7. UNAIDS. Terminology Guidelines. Available from: http://www. unaids.org/sites/default/files/media_ass et/2015_terminology_guidelines_en.pd f Accessed: October 2018

8. DeCarlo P, Ekstrand M. How does stigma affect HIV prevention and treatment? Available from: https://caps.ucsf.edu/wordpress/wpcontent/uploads/2016/11/StigmaFSUpd atefinal_20161020.pdf

9. UNAIDS Agenda for zero discrimination in health-care settings: 2017.

10. UNAIDS. Global HIV \& AIDS statistics - 2018 fact sheet: 2018. Available from: http://www.unaids.org/en/ resources/fact-sheet Accessed: October 2018

11. USAID. Available from: http://www. healthpolicyinitiative.com/ Accessed: October 2018

12. Olalekan AW, Akintunde AR, Olatunji MV. Perception of Societal Stigma and Discrimination Towards People Living with HIV/AIDS in Lagos, Nigeria: a Qualitative Study. Mater Socio-Medica 2014;26:191-4.

13. UNAIDS. Key human rights programmes to reduce stigma and discriminationand increase access to justice in national HIV responses. Available from: http://www.unaids.org/sites/default/file s/media_asset/Key_Human_Rights_Pro grammes_en_May2012_0.pdf Accessed: October 2018

14. Zamberia AM. HIV-related stigma and access to health care among people living with HIV in Swaziland. Dev South Afr 2011;28:669-80.

15. Zaidi MA, Griffiths R, Newson-Smith M, Levack W. Impact of stigma, culture and law on healthcare providers after occupational exposure to HIV and hepatitis C. Cult Health Sex 2012;14:37991.
16. Nyblade L, Stangl A, Weiss E, Ashburn $\mathrm{K}$. Combating HIV stigma in health care settings: what works? J Int AIDS Soc 2009;6:12-5.

17. African Gender and Media Initiative. Report on robbed of choice. Forced and coerced sterilization experiences of women living with HIV in Kenya; 2012 Available from: http://kelinkenya. org/wp-content/uploads/2010/10/ Report-on-Robbed-Of-Choice-Forcedand-Coerced-Sterilization-Experiencesof-Women-Living-with-HIV-inKenya.pdf

18. Banteyerga H, Kidanu A, Nyblade L, et al. Exploring HIV and AIDS stigma and related discrimination in Ethiopia: causes, manifestations, consequences, and coping mechanisms. Addis Ababa MizHasab Res Cent 2004:6-69.

19. Nyblade L, MacQuarrie K, Phillip F, et al. Measuring HIV stigma: results of a field test in Tanzania. Washington, DC: US Agency for International Development; 2005. Available at: https : / w w w. icrw. org/w p content/uploads/2016/10/WorkingReport-Measuring-HIV-StigmaResults-of-a-Field-Test-in-Tanzania.pdf

20. Mill J. Shrouded in Secrecy: Breaking the News of HIV Infection to Ghanaian Women. J Transcult Nurs 2003;14:6-16.

21. Ulasi CI, Preko PO, Baidoo JA, et al. HIV/AIDS-related stigma in Kumasi, Ghana. Health and Place 2009; 15:25562.

22. Awusabo-Asare K, Marfo C. Attitudes to and management of HIV/AIDS among health workers in Ghana: the case of Cape Coast municipality. Health Transit Rev 1997;7:271-280.

23. Government of Ghana. The Constitution of Ghana. Accra, Ghana: 1992.

24. Government of Ghana. National Guidelines for the Implementation of HIV Counselling and Testing in Ghana. Accra, Ghana: 2008.

25. Dawson-Amoah CG. Determinants of HIV Stigma among healthcare workers in Ghana. Doctoral dissertation, College of Health Science, Walden University, Minneapolis, MN: 2015. Available from: http://scholarworks. waldenu. edu/cgi/viewcontent.cgi article $=2530 \&$ context $=$ dissertations

26. Nyblade L, Jain A, Benkirane M, et al.
A brief, standardized tool for measuring HIV-related stigma among health facility staff: results of field testing in China, Dominica, Egypt, Kenya, Puerto Rico and St. Christopher \& Nevis. J Int AIDS Soc 2013:16:18718

27. Acquah SD, Dedzo M, AppiahDenkyira E, D'Almeida S. Ghana human resources for health country profile: 2011. Available from: ht tp://www.moh.gov.gh/w p content/uploads/ 2016/02/Ghana-hrhcountry-profile.pdf

28. Doka PJS, Danjin M, Dongs IS. HIV/AIDS-related stigma and discrimination among health-care providers in a tertiary health facility. J Med Sci 2017;37:44-49

29. Andrewin A, Chien L-Y. Stigmatization of patients with HIV/AIDS among doctors and nurses in Belize. AIDS Patient Care STDs 2008;22:897-906.

30. Famoroti TO, Fernandes L, Chima SC. Stigmatization of people living with HIV/AIDS by healthcare workers at a tertiary hospital in KwaZulu-Natal, South Africa: a cross-sectional descriptive study. BMC Med Ethics 2013; 14:S6.

31. Oe A. HIV related stigmatizing attitude and practice among health care workers in Northern Nigeria. J Infect Dis Immun 2011;3:226-32.

32. Holzemer WL, Makoae LN, Greeff M, et al. MeasuringHIV stigma for PLHAs and nurses over time in five African countries. Sahara-J 2009;6:76-82

33. Sayles JN, Ryan GW, Silver JS, et al. Experiences of social stigma and implications for healthcare among a diverse population of HIV positive adults. J Urban Health 2007;84:814-28.

34. Obi SN, Ifebunandu NA. Consequences of HIV testing without consent. Int J STD AIDS 2006;17:93-6.

35. Ekstrand ML, Ramakrishna J, Bharat S, Heylen E. Prevalence and drivers of HIV stigma among health providers in urban India: implications for interventions. J Int AIDS Soc 2013;16:18717

36. Health Policy Plus. Legal Audit Update on HIV Law and Policy in Ghana: A Summary of Developments in Ghanaian HIV and Human Rights Law Since 2010. Washington, DC: Palladium, Health Policy Plus 2017. 